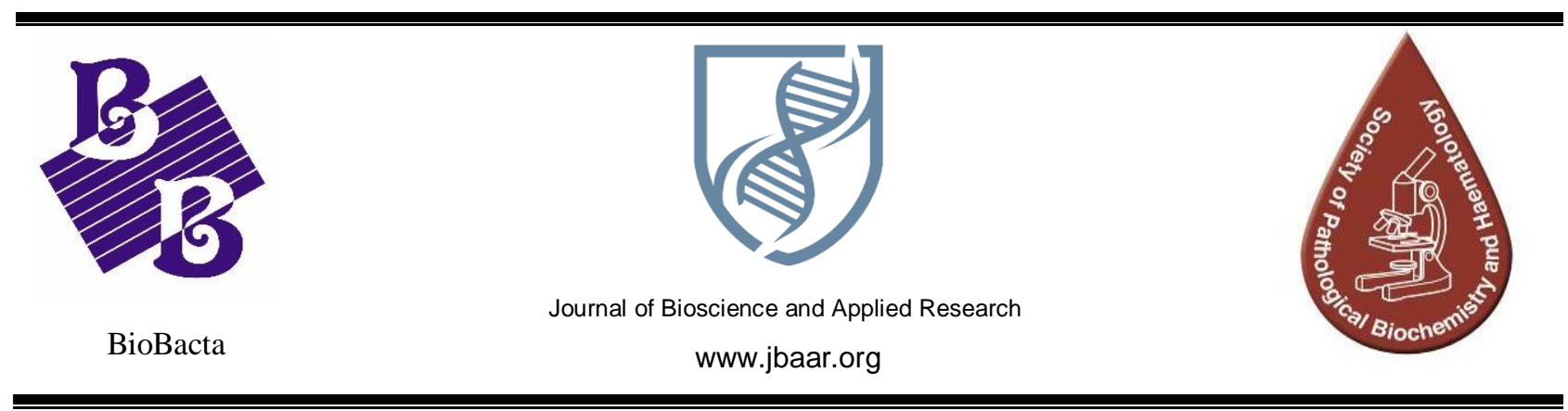

\title{
The efficiency of collagen III, metalloproteinase 1, carcinoembryonic antigen, and carbohydrate antigen 19.9 for colon cancer diagnosis
}

\author{
Abdelfattah M. Attallah', Mohamed A. El-Far', Mohamed M. Omran ${ }^{3}$, Mohamed A. \\ Abdelrazek ${ }^{1}$, Kareem A. Attallah', Mohamed S. Elbendary', Sara A. Soliman', \\ Rehab A. Atwa ${ }^{1}$ \\ ${ }^{1}$ Research \& Development Department, Biotechnology Research Center, New Damietta City, Egypt \\ ${ }^{2}$ Department of Chemistry, Faculty of Science, Mansoura University, Mansoura, Egypt \\ ${ }^{3}$ Department of Chemistry, Faculty of Science, Helwan University, Cairo, Egypt \\ * Corresponding author: Abdelfattah M. Attallah, PhD
}

Biotechnology Research Center, P.O. Box (14), 23 July St., Industrial Zone, New Damietta 34517, Egypt

Tel: 02/ 057 / 2429059 - 2429074, E-mail: amattallah@hotmail.com

DOI: $10.21608 /$ JBAAR. 2019.138673

\begin{abstract}
:
Background: Currently, blood markers are noninvasive methods for the diagnosis of patients with colorectal cancer (CRC). We evaluated four biomarkers (Carcinoembryonic antigen (CEA), carbohydrate antigen 19.9 (CA19.9), collagen III, and metalloproteinase 1 (MMP-1)) and their combination as a substitute method to enhance diagnosis of CRC. Subjects and methods: one hundred ninety-five patients who had undergone colonoscopy examination were included in the study (135 CRC and 60 benign growths). Also, 45 healthy individuals were included. Multivariate discriminant analysis (MDA) and area under the receiver operating characteristic curve (AUC) were applied for evaluating the diagnostic power of single and their combination. Results: levels of collagen III, CEA, and CA 19.9 increased while MMP-1 decreased with the progression of CRC (stages, positive lymph node invasion, distant organ metastasis, and high grades) with a significant difference $(P<0.01-P<0.0001)$. Combination of MMP-1, CEA, CA 19-9, and Collagen III yielded MC3 index had diagnostic power greater than every single marker could achieve alone. When differentiating colon cancer from benign growth, the AUC of MC3 index was 0.91 yielded $85.2 \%$ sensitivity and $86.7 \%$ specificity. Moreover, the MC3 index for patients with late stages, lymph node invasion, organ metastasis, and high-grade had AUC $=0.81,0.82,0.80$, and 0.80 higher than CEA and CA19.9. Conclusion: MC3 index is can be used as an effective index for early detection of CRC.
\end{abstract}

KEYWORDS: Colorectal cancer, Diagnosis, Blood markers, Extracellular matrix (ECM), Collagen, Metalloproteinase, Index, 


\section{INTRODUCTION}

$\mathrm{CRC}$ is a heterogeneous disease, there were about 1.8 million new cases of colorectal cancer in 2018 (Bray et al., 2018). CRC screening may be divided into two main categories: the first category includes fecal, blood, and urine biomarkers and the second group includes image-based methods such as flexible sigmoidoscopy and colonoscopy, (Dhaliwal et al., 2015). The restriction of fecal occult blood test (FOBT), it's being an indirect screening test. Sigmoidoscopy allows the physician to see the rectum and any tumor in the upper part of the colon will be not diagnosed (Jones et al., 2010). Colonoscopy is the gold standard method for colon cancer screening because colon cancer can be both detected and removed during the same procedure. But it is an invasive and not suitable method (Smith et al., 2012). Each of these methods has its shortcomings. Hence, a search for non-invasive convenient, and inexpensive tumor marker with a high degree of accuracy, is effective to monitor colon cancer (Torre et al., 2015). CEA and CA199 are routine tumor markers used in the screening of patients with colon cancer (Vukobrat-Bijedic et al., 2013). However, they are not suitable for the early detection of colon cancer, as their sensitivity and specificity are low (Lech et al., 2016). Thus, there is an urgent demand for biomarkers that can serve as diagnostic and prognostic markers for colon cancer (Hauptman and Glavac, 2017). Increased or decreased collagen levels are associated with CRC (Fang et al., 2014; Levental et al., 2009). Also, MMP-1 is an important proteolytic enzyme for extracellular matrix (ECM) remodeling during organ development and tissue regeneration (Alaaho and Kahari, 2005). MMP-1 is degraded II and III collagens that represent the main ingredients of the interstitial stroma (Said et al., 2014). Therefore, this work aimed to evaluate the diagnostic performances of CEA, CA 19-9, collagen III, and MMP-1 as single and combined markers associated with colon cancer clinicopathologic parameters.

\section{METHODOLOGY}

\section{Subjects}

Serum samples from 195 patients, 109 males and 86 females, with age ranged 22-79 years and Mean $\pm \mathrm{SD} ; 48.8 \pm 12.8$ years collected from the Oncology Center, Mansoura University Hospitals, Mansoura, Egypt between May 2016 and July 2018. They classified into 60 patients with benign growth conditions and 135 patients with colon cancer using colonoscopy. The tumor features were registered according to the Union for International Contrele Cancer-Tumor-Node-Metastasis (TNM) Staging System (Greene, 2003). Also, 45 healthy individuals (age- and sex-matched) were included. Neither of the patients with benign tumors nor of the healthy individuals had a record of any cancer. This study was approved by the ethics and scientific committees of Mansoura University Hospitals, Mansoura, Egypt. Informed consent was get from each individual to collaborate in the present study.

\section{Blood samples collection}

Serum samples were obtained after centrifuged blood samples at $4000 \mathrm{rpm}$ for 15 minutes. The serum was stocked at $-20{ }^{\circ} \mathrm{C}$ until use. CA19.9 and CEA were measured by ELISA assay (IRMA) (IBL, Germany) according to the industrial prescript.

\section{Determination of collagen III and MMP-1 using ELISA}

Collagen III and MMP-1 were determined using ELISA as described in our previous studies (Attallah et al., 2017). Collagen III and MMP-1 levels were expressed as $\mu / \mathrm{ml}$. Samples and controls were tested in duplicate. Samples were considered to be invalid and repeated the assay if the difference between levels $(\mu \mathrm{g} / \mathrm{ml})$ was more than $25 \%$. 
Statistical analysis: Data was expressed as mean \pm standard deviation (SD). Statistical analyses were carried out using Statistical Package for the Social Sciences (SPSS version, 24). ANOVA or t-test was used when the Kolmogorov-Smirnov test results were not significant, while the Mann-Whitney U test was used in cases with nonparametric variables. Significance is defined at $\mathrm{P}<0.05$. Novel indexes were developed using multivariate discriminant analysis and area under ROC. Diagnostic performances [sensitivity, specificity, positive predictive value, and negative predictive value] were determined after selections of the best cutoff points.

\section{RESULTS}

\section{Quantitation of collagen III and MMP-1 levels}

The 135 patients with colon cancer were presented according to tumor depth, lymph node invasion, and distant organ metastasis, and high grade. Levels of CEA, CA 19.9, and collagen III increased while MMP-1 decreased with late stages, positive lymph node invasion, distant organ metastasis, and high grades of colon cancer with a significant difference $(P<0.01-P<0.0001)$ as shown in Table 1 and Fig. 1.

Table 1: The level of candidate markers in different stages of the disease

\begin{tabular}{|c|c|c|c|c|}
\hline Groups & CA $19.9(\mathrm{U} / \mathrm{mL})$ & CEA (ng/mL) & MMP-1 $(\mu \mathrm{g} / \mathrm{ml})$ & $\begin{array}{c}\text { Collagen III } \\
(\mu \mathrm{g} / \mathrm{ml})\end{array}$ \\
\hline \multicolumn{5}{|c|}{ Benign growth $V s$ Colon cancer } \\
\hline Benign $(n=60)$ & $14.1 \pm 0.7$ & $4.0 \pm 0.3$ & $4.4 \pm 0.2$ & $3.1 \pm 0.5$ \\
\hline Cancer $(n=135)$ & $27.0 \pm 2.3$ & $19.4 \pm 2.7$ & $3.6 \pm 0.2$ & $11.0 \pm 0.8$ \\
\hline$P$ value & $<0.0001$ & $<0.0001$ & $<0.0001$ & $<0.0001$ \\
\hline \multicolumn{5}{|c|}{ Tumor depth (Early stages $V s$ Late stages) } \\
\hline Tis-T2 $(n=64)$ & $17.6 \pm 1.4$ & $10.4 \pm 2.4$ & $4.3 \pm 0.2$ & $8.1 \pm 0.8$ \\
\hline T3-T4 $(n=71)$ & $35.2 \pm 4.0$ & $27.7 \pm 4.7$ & $2.9 \pm 0.2$ & $12.8 \pm 1.2$ \\
\hline$P$ value & $<0.001$ & $<0.0001$ & $<0.0001$ & $<0.0001$ \\
\hline \multicolumn{5}{|c|}{ Lymph node invasion (N1-N2 Vs N0) } \\
\hline NO $(n=84)$ & $20.2 \pm 1.6$ & $11.2 \pm 2.2$ & $4.1 \pm 0.2$ & $8.5 \pm 0.6$ \\
\hline N1-N2 $(n=51)$ & $38.3 \pm 5.3$ & $32.8 \pm 6.0$ & $2.7 \pm 0.2$ & $15.1 \pm 1.6$ \\
\hline$P$ value & $<0.0001$ & $<0.0001$ & $<0.0001$ & $<0.0001$ \\
\hline \multicolumn{5}{|c|}{ Distant Organ Metastasis (M1 Vs M0) } \\
\hline M0 (n=99) & $21.4 \pm 1.7$ & $13.3 \pm 2.5$ & $3.8 \pm 0.1$ & $8.7 \pm 0.6$ \\
\hline M1 $(n=36)$ & $40.8 \pm 6.4$ & $34.4 \pm 6.7$ & $3.1 \pm 0.1$ & $16.8 \pm 1.9$ \\
\hline$P$ value & $<0.0001$ & $<0.0001$ & $<0.01$ & $<0.0001$ \\
\hline \multicolumn{5}{|c|}{ Tumor grade (Low-grade $V s$ High grade) } \\
\hline $\begin{array}{l}\text { Low grade }(n=80) \\
\text { G1-G2 }\end{array}$ & $23.1 \pm 2.6$ & $10.8 \pm 2.3$ & $3.9 \pm 0.2$ & $8.3 \pm 0.6$ \\
\hline $\begin{array}{l}\text { High grade }(n=55) \\
\text { G3-G4 }\end{array}$ & $32.5 \pm 4.3$ & $32.2 \pm 5.6$ & $3.0 \pm 0.1$ & $15.4 \pm 1.5$ \\
\hline$P$ value & $<0.05$ & $<0.0001$ & $<0.01$ & $<0.0001$ \\
\hline
\end{tabular}

Data presented as (Mean \pm SEM). SEM: Standard error of the mean, n: Number of samples $\boldsymbol{P}$ value $<0.05$ statistically significant difference. $\boldsymbol{P}$ value $<0.01$ statistically high significant difference. $\boldsymbol{P}$ value $<0.0001$ statistically extremely high significant difference. 

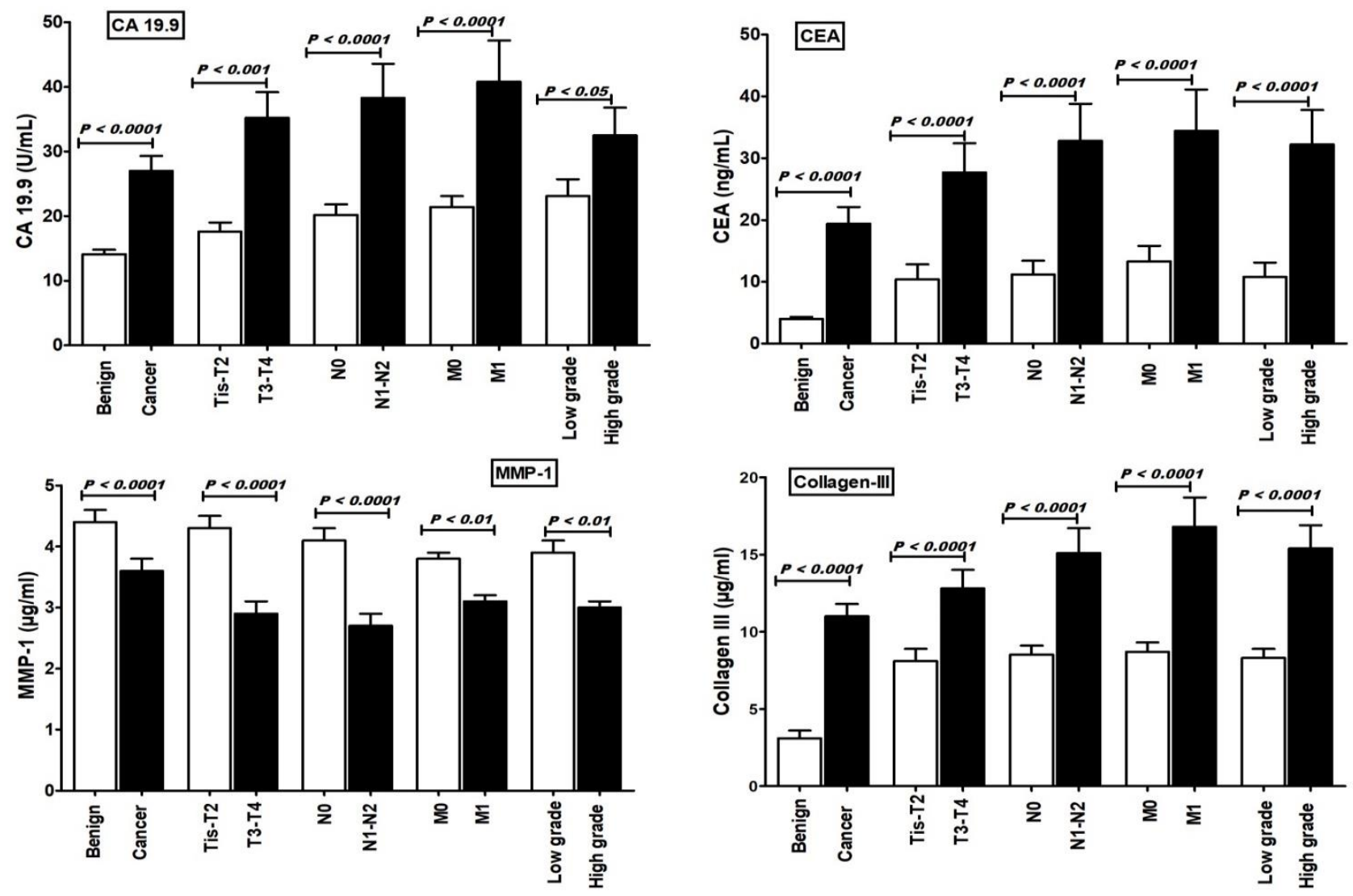

Figure 1: Levels of CA 19.9, CEA, MMP-1, and Collagen III in Benign growth vs Colon cancer, Tumor depth (early stages vs late stages), Lymph node invasion (N1-N2 vs N0) and Tumor grade (Low grade vs high grade).

\section{Diagnostic performance of candidate markers}

Using ROC curve analysis, we assessed and compared the diagnostic accuracy of CA 19.9, CEA, MMP-1, and collagen III in the diagnosis of colon cancer. The areas under ROC of candidate colon markers were in the order of CA 19.9 (0.58), CEA (0.61), MMP-1 (0.61), and collagen (0.84) to differentiate colon cancer from benign growth. Serum collagen was the most efficient marker for detecting patients with colon cancer $(\mathrm{AUC}=0.84)$, organ metastasis (AUC $=0.70$ ), and high grade from benign growth $(\mathrm{AUC}=0.69$ ). While MMP-1 was the most efficient marker for detecting patients with early-stage $(\mathrm{AUC}=0.72)$ and lymph node invasion (AUC=0.75). The diagnostic performance of candidate markers associated with colon cancer clinicopathologic parameters was listed in Table 2.

\section{Diagnostic performance of colon index}

The multiple discriminant analysis (MDA) showed that CA19.9, CEA, MMP-1, and collagen III 
were significantly $(P<0.0001)$ associated with colon cancer. Sex, age was not significantly $(P>0.05)$ associated with colon cancer. We developed a new index to differentiate between colon cancer patients and benign tumor named MC3 (MMP-1, CEA, CA19.9 and Collagen) index $=0.02 \times$ CEA $+0.02 \times$ CA19.9 + $0.14 \times$ Collagen - 0.2 x MMP-1. When differentiating colon cancer from benign growth, the AUC of MC3 index was 0.91 yielded $85.2 \%$ sensitivity and $86.7 \%$ specificity at 0.3 cutoffs. As shown in Fig. 2, the AUC of the MC3 index was 0.81, $0.82,0.80$, and 0.80 for patients with late stages, lymph node invasion, organ metastasis, and high grade. The diagnostic performances of the MC3 index associated with colon cancer clinicopathologic parameters were listed in Table 2. The diagnostic performances of the MC3 index were greater than every single marker could achieve alone.

Table 2: Diagnostic performance of CA 19.9, CEA, MMP-1, Collagen III, and Index

\begin{tabular}{|c|c|c|c|c|c|}
\hline Variables & Cut-off & Sensitivity & Specificity & PPV & NPV \\
\hline \multicolumn{6}{|c|}{ Benign growth $V s$ Colon cancer } \\
\hline CA $19.9(\mathrm{U} / \mathrm{mL})$ & 15.3 & $59.4 \%$ & $55.6 \%$ & $74.8 \%$ & $37.5 \%$ \\
\hline $\mathrm{CEA}(\mathrm{ng} / \mathrm{mL})$ & 3.0 & $62.2 \%$ & $60.0 \%$ & $77.8 \%$ & $41.4 \%$ \\
\hline MMP-1 $(\mu \mathrm{g} / \mathrm{mL})$ & 4.0 & $63.0 \%$ & $68.4 \%$ & $81.0 \%$ & $44.4 \%$ \\
\hline Collagen III $(\mu \mathrm{g} / \mathrm{mL})$ & 2.5 & $76.0 \%$ & $79.0 \%$ & $88.8 \%$ & $60.0 \%$ \\
\hline MC3 Index & 0.3 & $85.2 \%$ & $86.7 \%$ & $93.5 \%$ & $72.2 \%$ \\
\hline \multicolumn{6}{|c|}{ Tumor depth (Early stages $V s$ Late stages) } \\
\hline CA $19.9(\mathrm{U} / \mathrm{mL})$ & 15.3 & $60.7 \%$ & $64.8 \%$ & $59.7 \%$ & $65.7 \%$ \\
\hline $\mathrm{CEA}(\mathrm{ng} / \mathrm{mL})$ & 6.0 & $57.4 \%$ & $55.7 \%$ & $53.0 \%$ & $60.6 \%$ \\
\hline MMP-1 $(\mu \mathrm{g} / \mathrm{mL})$ & 4.0 & $73.8 \%$ & $74.6 \%$ & $71.4 \%$ & $76.8 \%$ \\
\hline Collagen III $(\mu \mathrm{g} / \mathrm{mL})$ & 6.2 & $73.2 \%$ & $73.8 \%$ & $70.7 \%$ & $75.5 \%$ \\
\hline MC3 Index & 1.5 & $82.0 \%$ & $82.3 \%$ & $79.4 \%$ & $84.0 \%$ \\
\hline \multicolumn{6}{|c|}{ Lymph node invasion (N1-N2 $V s$ N0) } \\
\hline CA $19.9(\mathrm{U} / \mathrm{mL})$ & 15.3 & $58.8 \%$ & $63.4 \%$ & $72.1 \%$ & $47.8 \%$ \\
\hline $\mathrm{CEA}(\mathrm{ng} / \mathrm{mL})$ & 6.0 & $68.6 \%$ & $68.0 \%$ & $78.1 \%$ & $56.4 \%$ \\
\hline MMP-1 $(\mu \mathrm{g} / \mathrm{mL})$ & 4.0 & $71.4 \%$ & $78.0 \%$ & $84.5 \%$ & $62.5 \%$ \\
\hline Collagen III $(\mu \mathrm{g} / \mathrm{mL})$ & 6.2 & $66.7 \%$ & $68.6 \%$ & $77.8 \%$ & $55.6 \%$ \\
\hline MC3 Index & 1.5 & $82.1 \%$ & $82.4 \%$ & $88.5 \%$ & $73.7 \%$ \\
\hline \multicolumn{6}{|c|}{ Distant Organ Metastasis (M1 Vs M0) } \\
\hline CA $19.9(\mathrm{U} / \mathrm{mL})$ & 15.3 & $66.7 \%$ & $61.6 \%$ & $76.7 \%$ & $48.8 \%$ \\
\hline CEA (ng/mL) & 6.0 & $70.0 \%$ & $67.0 \%$ & $78.3 \%$ & $56.5 \%$ \\
\hline $\operatorname{MMP}-1(\mu \mathrm{g} / \mathrm{mL})$ & 4.0 & $66.7 \%$ & $66.2 \%$ & $77.5 \%$ & $55.5 \%$ \\
\hline Collagen III $(\mu \mathrm{g} / \mathrm{mL})$ & 6.2 & $75.4 \%$ & $72.2 \%$ & $84.0 \%$ & $60.0 \%$ \\
\hline MC3 Index & 1.5 & $84.1 \%$ & $86.1 \%$ & $92.3 \%$ & $73.8 \%$ \\
\hline \multicolumn{6}{|c|}{ Tumor grade (Low-grade Vs High grade) } \\
\hline CA $19.9(\mathrm{U} / \mathrm{mL})$ & 15.3 & $66.2 \%$ & $60.0 \%$ & $70.0 \%$ & $56.0 \%$ \\
\hline $\mathrm{CEA}(\mathrm{ng} / \mathrm{mL})$ & 6.0 & $68.8 \%$ & $67.3 \%$ & $74.6 \%$ & $66.6 \%$ \\
\hline MMP-1 $(\mu \mathrm{g} / \mathrm{mL})$ & 4.0 & $66.2 \%$ & $65.5 \%$ & $73.0 \%$ & $63.0 \%$ \\
\hline Collagen III $(\mu \mathrm{g} / \mathrm{mL})$ & 6.2 & $70.0 \%$ & $67.0 \%$ & $77.8 \%$ & $67.6 \%$ \\
\hline MC3 Index & 1.5 & $81.5 \%$ & $83.8 \%$ & $86.5 \%$ & $72.7 \%$ \\
\hline
\end{tabular}

PPV: positive predictive value, NPV: negative predictive value 


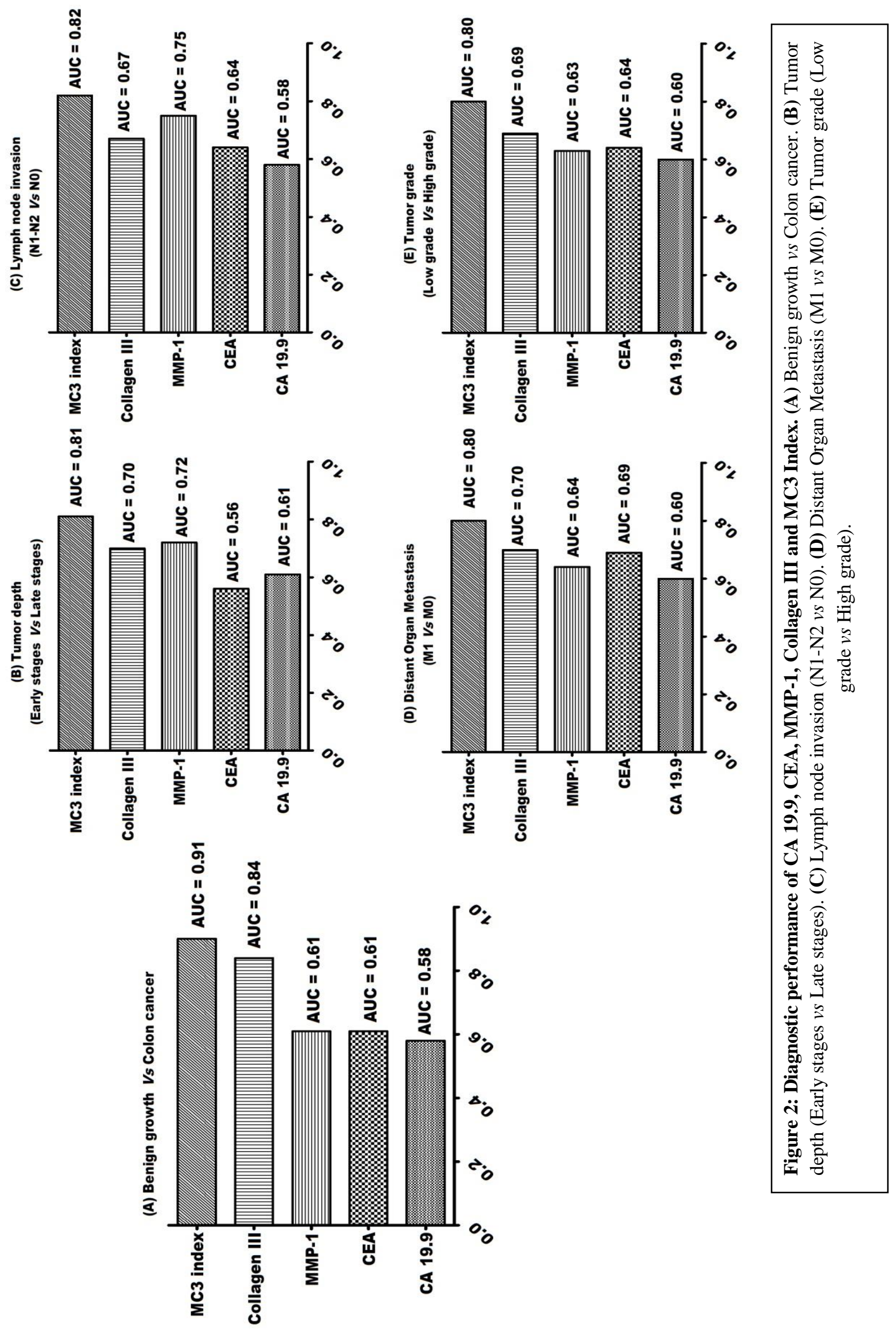




\section{DISCUSSION}

Colon cancer is a very common malignancy in the world (Araghi et al., 2018). Colon blood markers examinations are accepted in public than stool examination. The majority of participants in CRC screening choose blood examination $(83 \%)$ or stool examination (15\%) instead of colonoscopy (Adler et al., 2014). There is an urgent need for reliable blood markers or indexes to improve the diagnosis of colon cancer. MC3 index was based on four blood markers (MMP-1, collagen, CEA, and CA 19-9). These four candidate tests play a significant role in the development of colon cancer (Mysliwiec and Ornstein, 2002; Prager et al., 2014; Shibutani et al., 2014; Shiozawa et al., 2000; Walker et al., 2018). In this study, colon cancer patients with advanced stages, +ve lymph node invasion, metastasis, and high grades were correlated with higher levels of CEA, CA 19-9 and collagen III and decrease the level of MMP-1 in contrast to benign colon diseases with a significant difference $(P<0.01$ $P<0.0001)$. Cancer development associated with higher levels of collagen and other ECM molecules promotes cancer tissue rigidity (Walker et al., 2018). The elevated levels of collagen III in our patients with colon were agreed with the results of Basso et al (Basso et al., 2001) . MMPs play an important function in the development of human carcinoma. The destruction of ECM, tumor invasion, and metastasis were mediated with MMPs. MMPs also have angiogenic activity and play role in tumorigenesis (Mysliwiec and Ornstein, 2002). Shiozawa et al. (Shiozawa et al., 2000) reported that levels of MMP-1 were associated with the severity of CRC. CEA and CA 19-9 were used in diagnosing colon cancer (Shiozawa et al., 2000). CEA affects the formation of CRC by promoting tumor cell survival and encourage tumor angiogenesis (Prager et al., 2014). CA 19.9 plays important functions in a cell to cell recognition processes. The combination of CA 19.9 and CEA was used to diagnose patients with CRC (Shibutani et al., 2014). Our results similar to those reported by Cosimelli et al, who found that elevated levels of CEA and CA 19-9, correlated with the depth of invasion and pathological stage
(Cosimelli et al., 1989). CEA and CA19-9 have a low diagnostic performance (sensitivity of $40 \%-70 \%$ ) and (specificity of $73 \%-90 \%$ ) (Nikolaou et al., 2018). Epigenetic changes markers have been evaluated for colorectal diagnosis with 70\%-96\% sensitivity and $72 \%-96 \%$ specificity. DNA genetic and epigenetic biomarkers had sensitivity (30\% $87 \%$ ) and $96 \%$ specificity (Lech et al., 2016). Therefore, a combination of CEA, CA 19-9, collagen, and MMP-1 yielded a novel colon index. The AUC of colon index was 0.91 with $85.2 \%$ sensitivity and $86.7 \%$ specificity for differentiation of colon cancer from benign growths. Several authors combined single markers to raise the diagnostic performances for the diagnosis of colon cancer. The score based on six blood markers included CEA, ferritin, seprase, cytokeratin, osteopontin, and p53 gave sensitivity (70\%) and specificity (95\%) (Wild et al., 2010). MC3 index for patients with late stages, lymph node and organ metastasis had AUC $=0.81,0.82,0.80$ and 0.80 in comparison with $0.72,0.80$ and 0.80 for colon score (Attallah et al., 2018). The combination of serum lipids (cholesterol, high-density lipoprotein) and cancer antigens (CEA and CA19) were rose PPV to $88 \%$ for the diagnosis of colon cancer (Li et al., 2018). The AUC of $\mathrm{B} 7-\mathrm{H} 4$, osteopontin, tissue polypeptide-specific antigen, and CEA were 0.87 , $0.81,0.81$, and 0.83 , respectively. AUC of B7-H4 and CEA was 0.93 with high sensitivity (99\%) and $80 \%$ specificity for diagnosis CRC from healthy individuals (Wang et al., 2018).

In conclusion, a combination of serum CEA, CA 19-9, collagen, and MMP-1 yielded MC3 index with a diagnostic power greater than every single marker could achieve alone.

\section{REFERENCES}

Adler, A., Geiger, S., Keil, A., Bias, H., Schatz, P., deVos, T., Dhein, J., Zimmermann, M., Tauber, R., and Wiedenmann, B. (2014). Improving compliance to colorectal cancer screening using blood and stool-based tests in patients refusing screening colonoscopy in Germany. BMC Gastroenterol 14, 183. 
Ala-aho, R., and Kahari, V. M. (2005). Collagenases in cancer. Biochimie 87, 273-286.

Araghi, M., Soerjomataram, I., Jenkins, M., Brierley, J., and Morris, E. (2018). Global trends in colorectal cancer mortality: projections to the year 2035. Int J Cancer 2018.

Attallah, A. M., El-Far, M., Ghaly, M. F., Omran, M. M., Albannan, M. S., Attallah, A. A., Shoghey, T. M., Atrees, M. M., Elbendary, M. S., and Farid, K. (2017). Circulating levels of collagen III and MMP-1 in patients with chronic hepatitis $\mathrm{C}$ coinfected with hepatitis B virus. Br J Biomed Sci 74, 95-100.

Attallah, A. M., El-Far, M., Ibrahim, A. R., and ElDesouky, M. A. (2018). Clinical value of a diagnostic score for colon cancer based on serum CEA, CA199, cytokeratin-1 and mucin-1. Br J Biomed Sci 2018;75(3):122-127.

Basso, D., Belluco, C., Mazza, S., Greco, E., Della Rocca, F., Pauletto, P., Nitti, D., Lise, M., and Plebani, M. (2001). Colorectal cancer metastatic phenotype stimulates production by fibroblasts of $\mathrm{N}$ terminal peptide of type III collagen: clinical implications for prognosis. Clin Chim Acta 312, 135 142.

Bray, F., Ferlay, J., Soerjomataram, I., Siegel, R. L., Torre, L. A., and Jemal, A. (2018). Global cancer statistics 2018: GLOBOCAN estimates of incidence and mortality worldwide for 36 cancers in 185 countries. CA Cancer J Clin 68, 394-424.

Cosimelli, M., De Peppo, F., Castelli, M., Giannarelli, D., Schinaia, G., Castaldo, P., Buttini, G. L., Sciarretta, F., Bigotti, G., Di Filippo, F., et al. (1989). Multivariate analysis of a tissue CEA, TPA, and CA 19.9 quantitative study in colorectal cancer patients. A preliminary finding. Dis Colon Rectum 32, 389-397.

Dhaliwal, A., Vlachostergios, P. J., Oikonomou, K. G., and Moshenyat, Y. (2015). Fecal DNA testing for colorectal cancer screening: Molecular targets and perspectives. World J Gastrointest Oncol 2015;7(10):178-83.

Fang, M., Yuan, J., Peng, C., and Li, Y. (2014). Collagen as a double-edged sword in tumor progression. Tumour Biol 35, 2871-2882.
Greene, F. L. (2003). TNM staging for malignancies of the digestive tract: 2003 changes and beyond. Semin Surg Oncol 21, 23-29.

Hauptman, N., and Glavac, D. (2017). Colorectal Cancer Blood-Based Biomarkers. 2017, 2195361.

Jones, R. M., Devers, K. J., Kuzel, A. J., and Woolf, S. H. (2010). Patient-Reported Barriers to Colorectal Cancer Screening: A Mixed-Methods Analysis. American journal of preventive medicine 38, 508-516.

Lech, G., Slotwinski, R., Slodkowski, M., and Krasnodebski, I. W. (2016). Colorectal cancer tumour markers and biomarkers: Recent therapeutic advances. World J Gastroenterol 22, 1745-1755.

Levental, K. R., Yu, H., Kass, L., Lakins, J. N., Egeblad, M., Erler, J. T., Fong, S. F., Csiszar, K., Giaccia, A., Weninger, W., et al. (2009). Matrix crosslinking forces tumor progression by enhancing integrin signaling. Cell 139, 891-906.

Li, T., Qian, Y., Li, H., and Deng, J. (2018). Combination of serum lipids and cancer antigens as a novel marker for colon cancer diagnosis. Lipids Health Dis 17, 261.

Mysliwiec, A. G., and Ornstein, D. L. (2002). Matrix metalloproteinases in colorectal cancer. Clin Colorectal Cancer 1, 208-219.

Nikolaou, S., Qiu, S., Fiorentino, F., Rasheed, S., Tekkis, P., and Kontovounisios, C. (2018). Systematic review of blood diagnostic markers in colorectal cancer. Tech Coloproctol 22, 481-498.

Prager, G. W., Braemswig, K. H., Martel, A., Unseld, M., Heinze, G., Brodowicz, T., Scheithauer, W., Kornek, G., and Zielinski, C. C. (2014). Baseline carcinoembryonic antigen (CEA) serum levels predict bevacizumab-based treatment response in metastatic colorectal cancer. Cancer Sci 105, 996-1001.

Said, A. H., Raufman, J.-P., and Xie, G. (2014). The Role of Matrix Metalloproteinases in Colorectal Cancer. Cancers 6, 366-375.

Shibutani, M., Maeda, K., Nagahara, H., Ohtani, H., Sakurai, K., Toyokawa, T., Kubo, N., Tanaka, H., Muguruma, K., Ohira, M., et al. (2014). Significance of CEA and CA19-9 combination as a prognostic indicator and for recurrence monitoring in 
patients with stage II colorectal cancer. Anticancer Res 34, 3753-3758.

Shiozawa, J., Ito, M., Nakayama, T., Nakashima, M., Kohno, S., and Sekine, I. (2000). Expression of matrix metalloproteinase-1 in human colorectal carcinoma. Mod Pathol 13, 925-933.

Smith, A. P., Chiu, Y. S., and Lee, N. M. (2012). Towards universal screening for colon cancer: a cheap, reliable, noninvasive test using gene expression analysis of rectal swabs. ISRN Gastroenterol 2012, 170210.

Torre, L. A., Bray, F., Siegel, R. L., Ferlay, J., Lortet-Tieulent, J., and Jemal, A. (2015). Global cancer statistics, 2012. CA Cancer J Clin 65, 87-108. Vukobrat-Bijedic, Z., Husic-Selimovic, A., Sofic, A., Bijedic, N., Bjelogrlic, I., Gogov, B., and Mehmedovic, A. (2013). Cancer Antigens (CEA and
CA 19-9) as Markers of Advanced Stage of Colorectal Carcinoma. Med Arch 67, 397-401.

Walker, C., Mojares, E., and Del Rio Hernandez, A. (2018). Role of Extracellular Matrix in Development and Cancer Progression. Int J Mol Sci. 2018;19(10). pii: E3

Wang, P., Li, C., Zhang, F., Ma, X., and Gai, X. (2018). Clinical Value of Combined Determination of Serum B7-H4 with Carcinoembryonic Antigen, Osteopontin, or Tissue Polypeptide-Specific Antigen for the Diagnosis of Colorectal Cancer 2018, 4310790.

Wild, N., Andres, H., Rollinger, W., Krause, F., Dilba, P., Tacke, M., and Karl, J. (2010). A combination of serum markers for the early detection of colorectal cancer. Clin Cancer Res 16, 6111-6121. 